\title{
DYNAMICAL SUBSTITUTES OF THE LIBRATION POINTS FOR SIMPLIFIED SOLAR SYSTEM MODELS
}

\author{
G. GÓMEZ \\ IEEC E Departament de Matemàtica Aplicada i Anàlisi, \\ Universitat de Barcelona, Gran Via 585, 08007 Barcelona, Spain

\section{J.J. MASDEMONT and J.M. MONDELO} \\ IEEC $\&$ Departament de Matemàtica Aplicada I, \\ Universitat Politècnica de Catalunya E.T.S.E.I.B., Diagonal 647, 08028 \\ Barcelona, Spain

\begin{abstract}
The purpose of this paper is to develop a methodology to generate simplified models suitable for the analysis of the motion of a small particle, such as a spacecraft or an asteroid, in the Solar System. The procedure is based on applying refined Fourier analysis methods to the time-dependent functions that appear in the differential equations of the problem. The equations of the models obtained are quasi-periodic perturbations of the Restricted Three Body Problem that depend explicitly on natural frequencies of the Solar System. Some examples of these new models are given and compared with other ones found in the literature. For two of these new models, close to the Earth-Moon system, we have computed the dynamical substitutes of the collinear libration points.
\end{abstract}

\section{Introduction}

The main goal of this paper is the construction of quasi-periodic analytic models suitable for the study of the motion of a small particle in the Solar 
System. Without any simplification, the equations of the general problem form a set of 60 first order differential equations difficult to analyze. It is well known that very simple models, such as the Two Body Problem or the Restricted Three Body Problem (RTBP), are suitable for many purposes, since they give a good insight of dynamics in large regions of the phase space of the problem. Some of these models are restricted, which means that the small particle does not have any influence in the motion of the remaining bodies. The models introduced in this paper will be also restricted but not so simple as the ones already mentioned. We will try to keep within them the behavior of the dynamics related to the resonances between natural and excitation frequencies.

Most of the well known restricted problems take as starting point the RTBP. In our approach, instead of taking as starting equations those of the RTBP, we will consider Newton's equation for the motion of an infinitesimal body in the force field created by the bodies of the Solar System. Following the ideas of ${ }^{1}$, in Sect. 2 we introduce suitable reference systems and units such that, after selecting two bodies of the Solar System as primaries, the previously mentioned equations are set as a perturbation of the RTBP.

In Sect. 3, and for two particular choices of primaries, we perform the Fourier analysis of all the time periodic functions that appear in the new equations. In this way we are able to introduce a graded set of models with an increasing number of frequencies, that can be considered between the RTBP and the true equations. This is done in Sect. 4. Finally, in the last section we compute the dynamical substitutes of the collinear equilibrium points for two of the intermediate models introduced, close to the Earth-Moon system. The computation of the dynamical substitutes of the libration points in models different from the ones developed here can be found in $2,3,4,5$

Most of the results to be developed in this paper can be also found in ${ }^{6}$. This reference includes additional details about the development of models for the Earth-Moon system, which are not be given here. 


\section{General equations of motion}

Through the full paper, the set of bodies of the Solar System will be denoted by

$$
\mathcal{S}=\left\{P_{1}, \ldots, P_{11}\right\}
$$

where $P_{1}, \ldots, P_{11}$ are the nine planets, the Moon and the Sun, respectively. The mass of $P \in \mathcal{S}$ will be denoted by $m_{P}$.

Let us consider, in an intertial reference frame, Newton's equations for the motion of an infinitesimal body in the force field created by the bodies of the Solar System,

$$
\mathbf{R}^{\prime \prime}=G \sum_{i} m_{i} \frac{\left(\mathbf{R}_{i}-\mathbf{R}\right)}{\left\|\mathbf{R}-\mathbf{R}_{i}\right\|^{3}}
$$

were $G$ is the gravitational constant, $\mathbf{R}$ is the position of the infinitesimal body, $\mathbf{R}_{i}$ is the position of the Solar System body $i$ in the inertial system chosen and $m_{i}$ its mass. The associated Lagrangian is

$$
L\left(\mathbf{R}, \mathbf{R}^{\prime}, t^{*}\right)=\frac{1}{2}\left\langle\mathbf{R}^{\prime}, \mathbf{R}^{\prime}\right\rangle+\sum_{i \in \mathcal{S}} \frac{G m_{i}}{\left\|\mathbf{R}-\mathbf{R}_{i}\right\|},
$$

where $\mathbf{R}=(X, Y, Z)^{T}$ is the position of $Q$, the prime denotes the derivative with respect to time, $t^{*},\left\langle\mathbf{R}^{\prime}, \mathbf{R}^{\prime}\right\rangle$ is the dot product between $\mathbf{R}^{\prime}$ and $\mathbf{R}^{\prime}$, $G$ is the gravitational constant, $\mathbf{R}_{i}$ is the position of the body $i \in \mathcal{S}$ and $\|\cdot\|$ denotes the Euclidean norm. In practice, it is convenient that the reference frame and units, both in space and time, are consistent with the ephemerides data files used for the determination of $\mathbf{R}_{i}$.

Since we are interested in writing the equations of motion for $Q$ as a perturbation of the RTBP equations, we must select two bodies $I, J \in \mathcal{S}$ with $m_{I}>m_{J}$, which will play the role of primaries. In this way, the mass parameter, $\mu$, is defined as $\mu=m_{J} /\left(m_{I}+m_{J}\right)$, and so $1-\mu=$ $m_{I} /\left(m_{I}+m_{J}\right)$. Next, we must introduce the synodic reference frame. Recall that the origin of this system is set at the barycenter of $I, J$ and that the positions of the primaries are fixed at $(\mu, 0,0)$ and $(\mu-1,0,0){ }^{7}$.

Following Gómez et al. ${ }^{1}$, the transformation from synodical coordinates, $\mathbf{r}=(x, y, z)^{T}$, to sidereal ones, $\mathbf{R}$, is defined by

$$
\mathbf{R}=\mathbf{B}+k C \mathbf{r},
$$


where

- The translation, $\mathbf{B}$, is given by

$$
\mathbf{B}=\frac{m_{I} \mathbf{R}_{I}+m_{J} \mathbf{R}_{J}}{m_{I}+m_{J}},
$$

that clearly puts the barycenter of the primaries at the origin,

- The orthogonal matrix $C=\left(\mathbf{e}_{1}, \mathbf{e}_{2}, \mathbf{e}_{3}\right)$, sets the primaries on the $x^{-}$ axis and turns the instantaneous plane of motion of the primaries into the $x y$ plane (by requiring that the relative velocity of one primary with respect to the other has its third component equal to zero). The columns of $C$ are

$$
\mathbf{e}_{1}=\frac{\mathbf{R}_{J I}}{\left\|\mathbf{R}_{J I}\right\|}, \quad \mathbf{e}_{3}=\frac{\mathbf{R}_{J I} \times \mathbf{R}_{J I}^{\prime}}{\left\|\mathbf{R}_{J I} \times \mathbf{R}_{J I}^{\prime}\right\|}, \quad \mathbf{e}_{2}=\mathbf{e}_{3} \times \mathbf{e}_{1},
$$

being $\mathbf{R}_{J I}=\mathbf{R}_{I}-\mathbf{R}_{J}$.

- $k=\left\|\mathbf{R}_{J I}\right\|$ is a scaling factor which makes the distance between the primaries to be constant and equal to 1 .

It is important to remark that this change of variables is non-autonomous, since $\mathbf{B}, k$ and $C$ depend on time through the components of $\mathbf{R}_{I}$ and $\mathbf{R}_{J}$.

The change of coordinates given by Eq. (3) is checked to preserve the Lagrangian form of the equations and the new Lagrangian becomes

$$
\begin{aligned}
L\left(\mathbf{r}, \mathbf{r}^{\prime}, t^{*}\right)= & \frac{1}{2}\left\langle\mathbf{B}^{\prime}, \mathbf{B}^{\prime}\right\rangle+k^{\prime}\left\langle\mathbf{B}^{\prime}, \mathbf{s}\right\rangle+k\left\langle\mathbf{B}^{\prime}, \mathbf{s}^{\prime}\right\rangle \\
& +\frac{G m_{I}}{k\left[(x-\mu)^{2}+y^{2}+z^{2}\right]^{1 / 2}}+\frac{G m_{J}}{k\left[(x-\mu+1)^{2}+y^{2}+z^{2}\right]^{1 / 2}} \\
& +\sum_{i \in \mathcal{S}^{*}} \frac{G m_{i}}{k\left\|\mathbf{r}-\mathbf{r}_{i}\right\|}+\frac{1}{2} k^{\prime 2}\langle\mathbf{r}, \mathbf{r}\rangle+k k^{\prime}\left\langle\mathbf{s}, \mathbf{s}^{\prime}\right\rangle+\frac{1}{2} k^{2}\left\langle\mathbf{s}^{\prime}, \mathbf{s}^{\prime}\right\rangle,
\end{aligned}
$$

where $\mathbf{s}=C \mathbf{r}, \mathbf{r}_{i}$ is the position of the body $i$ in dimensionless coordinates and $\mathcal{S}^{*}$ represents the set of Solar System bodies without the two primaries $I, J$. To get the above expression of $L$, we use that $C$ defines an orthogonal transformation and, hence, it preserves the scalar product and the Euclidean norm.

Finally, we want to use the same time units as those usual for the RTBP, where $2 \pi$ time units correspond to one revolution of the primaries. If $t^{*}$ is 
some dynamical time and $n$ is the mean motion of $J$ with respect to $I$, then we perform the change of independent variable through

$$
t=n\left(t^{*}-t_{0}^{*}\right),
$$

where $t_{0}^{*}$ is a fixed epoch. From now on, $t$ will be called dimensionless time. In Table 2 we give the values of $n$ for the Earth-Moon and the Sun-(Earth+Moon) systems. In this second case, Earth+Moon means the Earth-Moon barycenter and, for this system, the Earth and the Moon are substituted in $\mathcal{S}$ by a fictitious body of mass $m_{E}+m_{M}$ behaving as their barycenter. The values in Table 2 are averaged values through the 6000 years covered by the JPL ephemerides file DE $406^{8}$, and have been computed from this file. Using Kepler's third law, $G\left(m_{I}+m_{J}\right)=n^{2} a^{3}$, we can also define the mean semi-major axis of the orbit of one primary around the other; these values are also given in Table 2 .

Table 1. Values for the mean motion and mean
semi-major axis used in the Earth-Moon and Sun-
(Earth+Moon) cases. The units are (Julian Days) ${ }^{-1}$ and
km.
\begin{tabular}{ccc}
\hline & Earth-Moon & Sun-(Earth+Moon) \\
\hline$n$ & 0.22997154619514 & 0.01720209883844 \\
$a$ & 384601.25606767 & 149598058.09228115 \\
\hline
\end{tabular}

If we denote with a dot the derivative with respect to $t$, remove those terms independent of $\mathbf{r}$ and $\dot{\mathbf{r}}$ and multiply by the scaling factor $a /\left(G\left(m_{I}+\right.\right.$ $\left.\left.m_{J}\right)\right)=1 /\left(n^{2} a^{2}\right)$, the new Lagrangian can be written as

$$
\begin{aligned}
L(\mathbf{r}, \dot{\mathbf{r}}, t)= & \frac{1}{a^{2}}\left(\dot{k}\langle\dot{\mathbf{B}}, \mathbf{s}\rangle+k\langle\dot{\mathbf{B}}, \dot{\mathbf{s}}\rangle+\frac{1}{2} \dot{k}^{2}\langle\mathbf{r}, \mathbf{r}\rangle+k \dot{k}\langle\mathbf{s}, \dot{\mathbf{s}}\rangle+\frac{1}{2} k^{2}\langle\dot{\mathbf{s}}, \dot{\mathbf{s}}\rangle\right) \\
& +\frac{a}{k}\left(\frac{1-\mu}{\left[(x-\mu)^{2}+y^{2}+z^{2}\right]^{1 / 2}}+\frac{\mu}{\left[(x-\mu+1)^{2}+y^{2}+z^{2}\right]^{1 / 2}}\right. \\
& \left.\quad+\sum_{i \in \mathcal{S}^{*}} \frac{\mu_{i}}{\left\|\mathbf{r}-\mathbf{r}_{i}\right\|}\right),
\end{aligned}
$$

where $\mu_{i}=m_{i} /\left(m_{I}+m_{J}\right)$.

Since $\mathbf{e}_{1}, \mathbf{e}_{2}, \mathbf{e}_{3}$ form an orthogonal basis, we have that $\left\langle\mathbf{e}_{i}, \mathbf{e}_{j}\right\rangle=\delta_{i j}$, $\left\langle\dot{\mathbf{e}}_{i}, \mathbf{e}_{j}\right\rangle=-\left\langle\mathbf{e}_{i}, \dot{\mathbf{e}}_{j}\right\rangle$ and $\left\langle\dot{\mathbf{e}}_{i}, \mathbf{e}_{i}\right\rangle=0$ for $i, j=1,2,3$. It can be further shown that $\left\langle\dot{\mathbf{e}}_{1}, \dot{\mathbf{e}}_{2}\right\rangle=0,\left\langle\dot{\mathbf{e}}_{2}, \dot{\mathbf{e}}_{3}\right\rangle=0$ and $\left\langle\dot{\mathbf{e}}_{1}, \mathbf{e}_{3}\right\rangle=0$. Recalling that $\mathbf{r}=(x, y, z)^{T}$, writing $\mathbf{s}=C \mathbf{r}=\mathbf{e}_{1} x+\mathbf{e}_{2} y+\mathbf{e}_{3} z$ and using the previous 
relations, we get

$$
\begin{aligned}
L(\mathbf{r}, \dot{\mathbf{r}}, t)= & a_{1}\left(\dot{x}^{2}+\dot{y}^{2}+\dot{z}^{2}\right)+a_{2}(x \dot{x}+y \dot{y}+z \dot{z})+a_{3}(x \dot{y}-\dot{x} y) \\
& +a_{4}(y \dot{z}-\dot{y} z)+a_{5} x^{2}+a_{6} y^{2}+a_{7} z^{2}+a_{8} x z \\
& +a_{9} \dot{x}+a_{10} \dot{y}+a_{11} \dot{z}+a_{12} x+a_{13} y+a_{14} z \\
& +a_{15}\left(\frac{1-\mu}{\left[(x-\mu)^{2}+y^{2}+z^{2}\right]^{1 / 2}}+\frac{\mu}{\left[(x-\mu+1)^{2}+y^{2}+z^{2}\right]^{1 / 2}}\right. \\
& \left.\quad+\sum_{i \in \mathcal{S}^{*}} \frac{\mu_{i}}{\left[\left(x-x_{i}\right)^{2}+\left(y-y_{i}\right)^{2}+\left(z-z_{i}\right)^{2}\right]^{1 / 2}}\right),
\end{aligned}
$$

where the $a_{i}$ are time dependent functions that can be computed in terms of the positions, velocities, accelerations and over-accelerations of the two primaries. Using Lagrange equations $(d(\partial L / \partial \dot{\mathbf{r}}) / d t=\partial L / \partial \mathbf{r})$ we get the second-order differential equations

$$
\left\{\begin{array}{l}
\ddot{x}=b_{1}+b_{4} \dot{x}+b_{5} \dot{y}+b_{7} x+b_{8} y+b_{9} z+b_{13} \frac{\partial \Omega}{\partial x}, \\
\ddot{y}=b_{2}-b_{5} \dot{x}+b_{4} \dot{y}+b_{6} \dot{z}-b_{8} x+b_{10} y+b_{11} z+b_{13} \frac{\partial \Omega}{\partial y}, \\
\ddot{z}=b_{3}-b_{6} \dot{y}+b_{4} \dot{z}+b_{9} x-b_{11} y+b_{12} z+b_{13} \frac{\partial \Omega}{\partial z},
\end{array}\right.
$$

being

$$
\begin{aligned}
\Omega= & \frac{1-\mu_{I, J}}{\sqrt{\left(x-\mu_{I, J}\right)^{2}+y^{2}+z^{2}}}+\frac{\mu_{I, J}}{\sqrt{\left(x-\mu_{I, J}+1\right)^{2}+y^{2}+z^{2}}} \\
& +\sum_{\substack{j \in \mathcal{S} \\
j \neq I, J}} \frac{\mu_{I, J, j}}{\sqrt{\left(x-x_{j}\right)^{2}+\left(y-y_{j}\right)^{2}+\left(z-z_{j}\right)^{2}}}
\end{aligned}
$$

where $\mu_{I, J, j}=\frac{m_{j}}{m_{I}+m_{J}}$ and the $b_{i}$ time-dependent functions are defined as

$$
\begin{aligned}
& b_{1}=\frac{-1}{k}\left(\ddot{\mathbf{B}} \mathbf{e}_{1}\right), \quad b_{6}=2\left(\dot{\mathbf{e}}_{2} \mathbf{e}_{3}\right), \quad b_{11}=\frac{2 \dot{k}}{k}\left(\dot{\mathbf{e}}_{2} \mathbf{e}_{3}\right)+\left(\ddot{\mathbf{e}}_{2} \mathbf{e}_{3}\right), \\
& b_{2}=\frac{-1}{k}\left(\ddot{\mathbf{B}} \mathbf{e}_{2}\right), \quad b_{7}=\left(\dot{\mathbf{e}}_{1} \dot{\mathbf{e}}_{1}\right)-\frac{\ddot{k}}{k}, \quad b_{12}=\left(\dot{\mathbf{e}}_{3} \dot{\mathbf{e}}_{3}\right)-\frac{\ddot{k}}{k}, \\
& b_{3}=\frac{-1}{k}\left(\ddot{\mathbf{B}} \dot{\mathbf{e}}_{3}\right) \quad b_{8}=\frac{2 \dot{k}}{k}\left(\dot{\mathbf{e}}_{1} \mathbf{e}_{2}\right)+\left(\ddot{\mathbf{e}}_{1} \mathbf{e}_{2}\right), \quad b_{13}=\frac{a^{3}}{k^{3}}, \\
& b_{4}=\frac{-2 \dot{k}}{k} \quad b_{9}=\left(\dot{\mathbf{e}}_{1} \dot{\mathbf{e}}_{3}\right), \\
& b_{5}=2\left(\dot{\mathbf{e}}_{1} \mathbf{e}_{2}\right), \quad b_{10}=\left(\dot{\mathbf{e}}_{2} \dot{\mathbf{e}}_{2}\right)-\frac{\ddot{k}}{k} \text {. }
\end{aligned}
$$


We note that setting $b_{i}=0$ for $i \neq 5,7,10,13, b_{5}=2, b_{7}=b_{10}=b_{13}=1$ and skipping the sum over $\mathcal{S}^{*}$ in Eq. (6), the Eqs. (5) become the usual RTBP equations with mass parameter $\mu$. Therefore, we can see Eqs. (5) as a perturbation of the RTBP equations. Once the primaries have been fixed, we will get an idea of the order of magnitude of the perturbation, by looking at the first coefficient of the Fourier expansions of the $b_{i}$ functions. The Fourier analysis of this functions will be done in the next sections for two different systems.

If we we introduce momenta as

$$
p_{x}=\frac{\partial L}{\partial \dot{x}}, \quad p_{y}=\frac{\partial L}{\partial \dot{y}}, \quad p_{z}=\frac{\partial L}{\partial \dot{z}},
$$

we have

$$
\begin{aligned}
& p_{x}=2 a_{1} \dot{x}+a_{2} x-a_{3} y+a_{9}, \\
& p_{y}=2 a_{1} \dot{y}+a_{2} y+a_{3} x-a_{4} z+a_{10}, \\
& p_{z}=2 a_{1} \dot{z}+a_{2} z+a_{4} y+a_{11} .
\end{aligned}
$$

It is known that, in this case, the Hamiltonian of the model is given in terms of the Lagrangian as $H(\mathbf{r}, \mathbf{p}, t)=\dot{x} p_{x}+\dot{y} p_{y}+\dot{z} p_{z}-L(\mathbf{r}, \dot{\mathbf{r}}, t)$, where $\mathbf{p}=\left(p_{x}, p_{y}, p_{z}\right)^{T}$ and $\dot{x}, \dot{y}, \dot{z}$ can be written in terms of $p_{x}, p_{y}, p_{z}$ from (8). After expanding the previous expression of $H$, skipping terms that do not depend on $\mathbf{r}, \mathbf{p}$ and collecting we obtain

$$
\begin{aligned}
H(\mathbf{r}, \mathbf{p}, t)= & c_{1}\left(p_{x}^{2}+p_{y}^{2}+p_{z}^{2}\right)+c_{2}\left(x p_{x}+y p_{y}+z p_{z}\right)+c_{3}\left(y p_{x}-x p_{y}\right)+ \\
& +c_{4}\left(z p_{y}-y p_{z}\right)+c_{5} x^{2}+c_{6} y^{2}+c_{7} z^{2}+c_{8} x z+ \\
& +c_{9} p_{x}+c_{10} p_{y}+c_{11} p_{z}+c_{12} x+c_{13} y+c_{14} z+ \\
& +c_{15}\left(\frac{1-\mu}{\left[(x-\mu)^{2}+y^{2}+z^{2}\right]^{1 / 2}}+\frac{\mu}{\left[(x-\mu+1)^{2}+y^{2}+z^{2}\right]^{1 / 2}}+\right. \\
& \left.\quad+\sum_{i \in \mathcal{S}^{*}} \frac{\mu_{i}}{\left[\left(x-x_{i}\right)^{2}+\left(y-y_{i}\right)^{2}+\left(z-z_{i}\right)^{2}\right]^{1 / 2}}\right),
\end{aligned}
$$


where

$$
\begin{aligned}
c_{1} & =\frac{a^{2}}{2 k^{2}}, & c_{9} & =\frac{-1}{k} \dot{\mathbf{B}}_{1}, \\
c_{2} & =\frac{-k}{k}, & c_{10} & =\frac{-1}{k} \dot{\mathbf{B}}_{2}, \\
c_{3} & =\dot{\mathbf{e}}_{1} \mathbf{e}_{2}, & c_{11} & =\frac{-1}{k} \dot{\mathbf{B}}_{3}, \\
c_{4} & =\dot{\mathbf{e}}_{2} \mathbf{e}_{3}, & c_{12} & =\frac{k}{a^{2}}\left(\left(\dot{\mathbf{e}}_{1} \mathbf{e}_{2}\right)\left(\dot{\mathbf{B}}_{2}\right)-\dot{\mathbf{B}} \dot{\mathbf{e}}_{1}\right), \\
c_{5} & \left.=\frac{k^{2}}{2 a^{2}}\left(\left(\dot{\mathbf{e}}_{1} \mathbf{e}_{2}\right)^{2}-\dot{\mathbf{e}}_{1} \dot{\mathbf{e}}_{1}\right)\right), & c_{13} & =\frac{k}{a^{2}}\left(\left(\dot{\mathbf{e}}_{2} \mathbf{e}_{3}\right)\left(\dot{\mathbf{B}} \mathbf{e}_{3}\right)\right. \\
c_{6} & =\frac{k^{2}}{2 a^{2}}\left(\left(\dot{\mathbf{e}}_{1} \mathbf{e}_{2}\right)^{2}+\left(\dot{\mathbf{e}}_{2} \mathbf{e}_{3}\right)^{2}-\dot{\mathbf{e}}_{2} \dot{\mathbf{e}}_{2}\right), & & \left.-\left(\dot{\mathbf{e}}_{1} \mathbf{e}_{2}\right)\left(\dot{\mathbf{B}} \mathbf{e}_{1}\right)-\dot{\mathbf{B}} \dot{\mathbf{e}}_{2}\right), \\
c_{7} & =\frac{k^{2}}{2 a^{2}}\left(\left(\dot{\mathbf{e}}_{2} \mathbf{e}_{3}\right)^{2}-\dot{\mathbf{e}}_{3} \dot{\mathbf{e}}_{3}\right), & c_{14} & =\frac{-k}{a^{2}}\left(\left(\dot{\mathbf{e}}_{2} \mathbf{e}_{3}\right)\left(\dot{\mathbf{B}} \mathbf{e}_{2}\right)+\dot{\mathbf{B}} \dot{\mathbf{e}}_{3}\right), \\
c_{8} & =\frac{k^{2}}{a^{2}}\left(-\left(\dot{\mathbf{e}}_{1} \mathbf{e}_{2}\right)\left(\dot{\mathbf{e}}_{2} \mathbf{e}_{3}\right)-\dot{\mathbf{e}}_{1} \dot{\mathbf{e}}_{3}\right), & c_{15} & =\frac{-a}{k}
\end{aligned}
$$

Note that setting $c_{1}=1 / 2, c_{3}=1, c_{15}=1$, the rest of $c_{i}$ equal to zero, and skipping the sum over $\mathcal{S}^{*}$, we recover the Hamiltonian of the RTBP ${ }^{7}$. The Hamiltonian of the bicircular coherent models ${ }^{2,4}$ can also be recovered by setting $c_{1}=\alpha_{1} / 2, c_{2}=\alpha_{2}, c_{3}=\alpha_{3}, c_{12}=\alpha_{4}, c_{13}=\alpha_{5}, c_{15}=-\alpha_{6}$, the rest of $c_{i}$ equal to zero, and letting the sum over $\mathcal{S}^{*}$ run only over the Sun, with $x_{S}=\alpha_{7}, y_{S}=\alpha_{8}$ and $z_{S}=0$.

\section{Fourier analysis}

This section is devoted to the results of the Fourier analysis applied to all the time-dependent functions appearing in Eqs. (5), (6), this is: the $b_{j}$ functions and the coordinates, $x_{i}, y_{i}, z_{i}$, of the bodies of the Solar System included in $\mathcal{S}^{*}$. The Fourier analysis follows the methodology developed in Gmez et $a l .{ }^{9}$, which is a refined procedure that allows a very accurate determination of frequencies and amplitudes for analytic quasi-periodic functions. Here we will discuss the selection of the main parameters used in the method as well as the results obtained. Although the analysis can be done for any set of primaries, we have selected two different couples - the Earth and 
the Moon and the Sun and the Earth-Moon barycenter- because of their relevance in many spacecraft mission analysis simulations.

\subsection{Fourier analysis of the $b_{i}$ functions}

Using the algorithm described in ${ }^{9}$, we have performed Fourier analysis of the $\left\{b_{i}\right\}_{i=1, \ldots, 13}$ functions, both for the Earth-Moon case and the Sun(Earth+Moon) case. This means that for each $b_{i}$ function we have obtained a set of frequencies and amplitudes that define its quasi-periodic approximation as a trigonometric polynomial, $Q_{b_{i}}(t)$. As for any Fourier procedure, the most relevant parameters to be specified are the size, $T$, of the time (sampling) interval and the number, $N$, of equally spaced sampling points chosen in the interval. These parameters define, for instance, the Nyquist critical frequency, $\omega_{c}=N /(2 T)$, that fixes the window within we will find all the frequencies (true or aliased) of our time series. So, the first thing that we need is some criteria to choose properly $T$ and $N$.

Due to our implementation of the Fourier analysis procedure, the parameter $N$ must range over powers of two. For consistency, the length, $T$, of the time-interval has also been chosen to range over a geometric progression, and the time-interval has always started at January 1st, 2001. The smallest time-interval length, $T_{\min }$, has been taken of 95 years $(34698.75$ Julian days) and the greatest time-interval length, $T_{\max }$, has been chosen as the maximum time-interval covered by the JPL DE406 ephemerides after Jan 1st 2001, which is 364938 Julian days (999.15 years). Therefore, we have let $T$ range over the set $\left\{\delta^{n} T_{\min }\right\}_{n=0}^{10}$ where $\delta=\left(T_{\max } / T_{\min }\right)^{1 / 10}$. The time units used are revolutions of the secondary $(J)$ around the primary $(I)$ or, equivalently, dimensionless time divided by $2 \pi$. The reason for this is that, in this way, the frequency 1.0 corresponds to one revolution of $J$ around $I$, which has a more intuitive meaning (one lunar month in the Earth-Moon case, one sidereal year in the Sun-(Earth+Moon) case) that will help in the elaboration of the intermediate models of motion. Moreover, in order to evaluate the trigonometric approximations of the $b_{i}$ functions, we only have to multiply the frequencies found by the dimensionless time, without the need of an additional $2 \pi$ factor.

The maximum number of samples $N_{\max }$ has been chosen to be $2^{20}$, in order to allow for "comfortable" runs on machines with $64 \mathrm{MB}$ of memory 
(or, equivalently, bi-processor machines with 128MB). For each value of $T$, the minimum number of samples has been chosen such that $\frac{N}{2 T} \geq 1.5$, in order to make the maximum detectable frequency to be at least 1.5.

Assume that, for certain fixed values of $T$ and $N$, we have performed Fourier analysis of a given function $b_{i}(t)$ obtaining the trigonometric polynomial $Q_{b_{i}}(t)$. Then, we can easily compute the maximum difference between the analyzed function and its quasi-periodic approximation at the sampling points, that is,

$$
d_{\max }=\max _{l=0, \ldots, N-1}\left|b_{i}\left(t_{l}\right)-Q_{b_{i}}\left(t_{l}\right)\right|,
$$

where $t_{l}=l(T / N), l=0, \ldots, N-1$ are the sampling epochs. In Figure 1 (Sun-(Earth+Moon) case, see ${ }^{6}$ for the Earth-Moon one) we have represented, for all the $b_{i}$ functions, the minimum of $d_{\max }$ with respect to the different values of $N$ explored, when varying $T$ according to the preceding discussion.

To reduce the leakage effect, in all the computations we have multiplied our data by a Hanning function of order two

$$
H_{T}^{2}(t)=\frac{2}{3}\left(1-\cos \left(2 \pi \frac{t}{T}\right)\right)^{2} .
$$

The advantages of the Hanning function with respect to other well-known window functions ${ }^{10}$ are its simplicity and its degree of differentiability. For instance, $H_{T}^{n}(t)$ has degree $2 n$, whereas a general "triangle window function" $T_{T}^{n}(t)$ has degree just $n$. This higher degree of regularity implies a faster decay of the Fourier coefficients (see ${ }^{9}$ for more details).

In order to control aliasing, two different strategies have been followed. The first one is based on time-domain, and consists in computing the maximum difference between the initial function and its quasi-periodic approximation, over a refinement of the initial grid of data used for the Fourier analysis. This difference will be denoted as $\alpha_{1}$. If it increases significantly when increasing the number of points of the grid, then aliasing is very likely to occur. For this test, we have used a refinement with $16 \mathrm{~N}$ equally spaced points in $[0, T]$.

The second anti-aliasing strategy is based on frequency-domain. It consists in computing the number of rightmost consecutive harmonics of the residual Discrete Fourier Transform (DFT) that have modulus less than a 

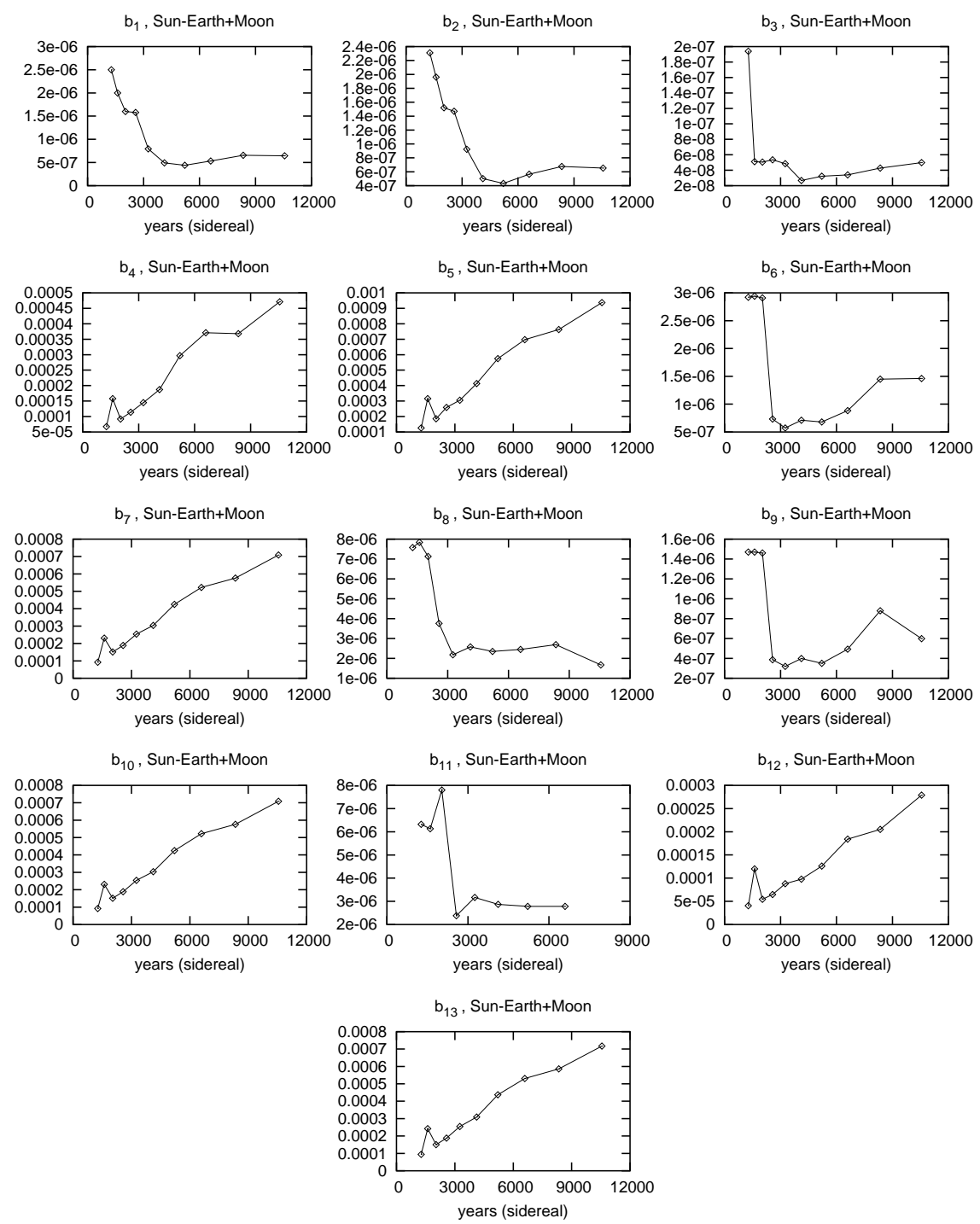

Fig. 1. Error results of the Fourier analysis of the $b_{i}$ functions in the Sun-(Earth+Moon) case. For each value of $T$ explored, we have represented the minimum value of $d_{\text {max }}$ with respect to $N$. The values of $T$ are given in sidereal years (revolutions of the Earth around the Sun). 
fraction of the maximum modulus of the residual DFT. Then, we divide this number by $N / 2$, the total number of harmonics, and this defines the parameter $\alpha_{2}$. That is, if $E_{i}(t)=b_{i}(t)-Q_{b_{i}}(t)$ is the error of the trigonometric approximation of $b_{i}(t)$ and $c_{E_{i}, T, N}(j), s_{E_{i}, T, N}(j), j=0, \ldots, N / 2$, are the cosine and sine coefficients of its DFT, we compute

$$
\begin{aligned}
p_{E_{i}, T, N}(j) & =\left(\left(c_{E_{i}, T, N}(j)\right)^{2}+\left(s_{E_{i}, T, N}(j)\right)^{2}\right)^{1 / 2}, \\
p_{\max } & =\max _{j=0, \ldots, N / 2} p_{E_{i}, T, N}(j), \\
\alpha_{2} & =\frac{\min \left\{j: p_{E_{i}, T, N}(l) \leq p_{\max } / 25 \text { for } l=j, \ldots, N / 2\right\}}{N / 2}
\end{aligned}
$$

Then, for instance, a value of 0.2 for $\alpha_{2}$ means that there are no frequencies greater than $0.8 \cdot \omega_{\max }=0.8 \cdot(N / 2 T)$, with amplitude greater than $1 / 25$ times the modulus of the residual DFT, so we do not expect aliasing in the corresponding Fourier analysis. We are assuming here that amplitudes decrease as frequencies increase, which is ensured by the Cauchy estimates of the Fourier coefficients for an analytic quasi-periodic function.

As an example of aliasing and how the two previously-described strategies detect it, we have represented in Figure 2 the residual DFT of some of the Fourier analysis of the $b_{1}$ function in the Earth-Moon case. Some numerical values of these analysis are given in Table 3.1. In the left plot, we see that for $N=16384$ there are frequencies of high amplitude near $\omega_{\max }=4.02903$. As we increase $N$, the amplitude of the frequencies near $\omega_{\max }$ decrease and the values of $d_{\max }$ as well as the parameter $\alpha_{1}$ of the first anti-aliasing strategy become closer.
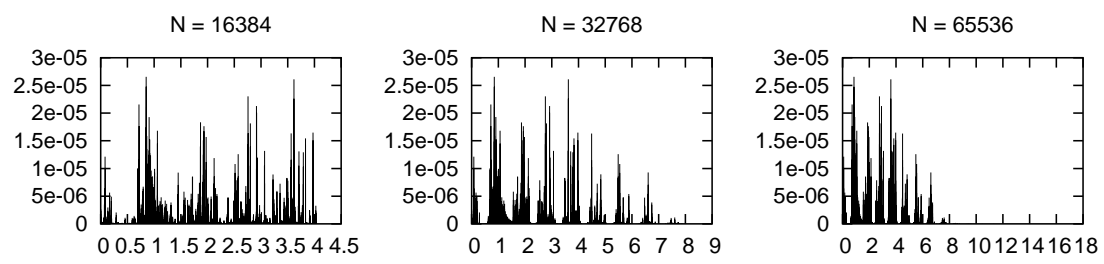

Fig. 2. Modulus of the residual DFT some of the Fourier analysis of the $b_{1}$ function in the Earth-Moon case. The values of the parameters of these analysis are given in Table 3.1.

According to this, for the results displayed in Figure 1 only those analyses with $\alpha_{1}<1.2 d_{\max }$ and $\alpha_{2} \geq 0.2$ have been taken into account. 
For the generation of simplified models for the Solar System, among all the analysis performed we have selected the best ones in terms of minimum $d_{\max }$. The corresponding parameters of these "best" analysis are given in Tables 3.1 (Earth-Moon) and 3.1 (Sun-(Earth+Moon)).

Table 2. Parameters associated to the Fourier analysis of Fig. 2. From left to

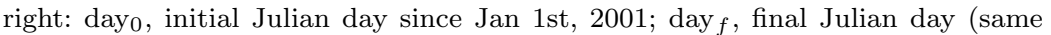
units); $T$, time interval in $J$-revolutions; $N$, number of points used; $\omega_{\max }$, maximum detectable frequency; $p_{\max }$, maximum modulus of the residual DFT; $d_{\max }$, maximum difference between $b_{1}$ and its quasi-periodic approximation over the samples; $\alpha_{1}, \alpha_{2}$, values of the two anti-aliasing parameters.

\begin{tabular}{ccccccccc}
\hline day $_{0}$ & day $_{f}$ & $T$ & $N$ & $\omega_{\max }$ & $p_{\max }$ & $d_{\max }$ & $\alpha_{1}$ & $\alpha_{2}$ \\
\hline 366 & 55917.4 & 2033.24 & 16384 & 4.02903 & $2.66 \mathrm{E}-05$ & $4.90 \mathrm{E}-04$ & $2.29 \mathrm{E}-03$ & 0.0007 \\
366 & 55917.4 & 2033.24 & 32768 & 8.05806 & $2.66 \mathrm{E}-05$ & $5.30 \mathrm{E}-04$ & $5.67 \mathrm{E}-04$ & 0.1633 \\
366 & 55917.4 & 2033.24 & 65536 & 16.1161 & $2.66 \mathrm{E}-05$ & $5.63 \mathrm{E}-04$ & $5.67 \mathrm{E}-04$ & 0.5816 \\
\hline
\end{tabular}

Table 3. Values of the parameters for the best Fourier analyses of the $b_{i}$ functions for the Earth-Moon case.

\begin{tabular}{cccccc}
\hline function & $T$ (days) & $T$ (J-rev.) & $N$ & $p_{\max }$ & $d_{\max }$ \\
\hline$b_{1}$ & 55551.4 & 2033.24 & 65536 & $2.66 \mathrm{E}-05$ & $5.63 \mathrm{E}-04$ \\
$b_{2}$ & 55551.4 & 2033.24 & 65536 & $2.67 \mathrm{E}-05$ & $5.49 \mathrm{E}-04$ \\
$b_{3}$ & 55551.4 & 2033.24 & 32768 & $3.30 \mathrm{E}-06$ & $5.58 \mathrm{E}-05$ \\
$b_{4}$ & 55551.4 & 2033.24 & 65536 & $2.31 \mathrm{E}-06$ & $5.01 \mathrm{E}-05$ \\
$b_{5}$ & 43904.0 & 1606.94 & 32768 & $4.85 \mathrm{E}-06$ & $9.16 \mathrm{E}-05$ \\
$b_{6}$ & 70288.7 & 2572.64 & 32768 & $3.92 \mathrm{E}-08$ & $1.13 \mathrm{E}-06$ \\
$b_{7}$ & 55551.4 & 2033.24 & 65536 & $3.51 \mathrm{E}-06$ & $7.81 \mathrm{E}-05$ \\
$b_{8}$ & 55551.4 & 2033.24 & 524288 & $1.96 \mathrm{E}-07$ & $5.94 \mathrm{E}-06$ \\
$b_{9}$ & 70288.7 & 2572.64 & 65536 & $1.97 \mathrm{E}-08$ & $5.69 \mathrm{E}-07$ \\
$b_{10}$ & 55551.4 & 2033.24 & 65536 & $3.51 \mathrm{E}-06$ & $7.83 \mathrm{E}-05$ \\
$b_{11}$ & 70288.7 & 2572.64 & 65536 & $1.67 \mathrm{E}-08$ & $5.05 \mathrm{E}-07$ \\
$b_{12}$ & 43904.0 & 1606.94 & 32768 & $1.58 \mathrm{E}-06$ & $3.29 \mathrm{E}-05$ \\
$b_{13}$ & 55551.4 & 2033.24 & 65536 & $3.51 \mathrm{E}-06$ & $7.99 \mathrm{E}-05$ \\
\hline
\end{tabular}

\subsection{Fourier analysis of the positions of the planets}

In order to complete the quasi-periodic approximation of all the timedependent part in the vector-field given by Eqs. (5), we have performed Fourier analysis of each coordinate $x_{i}, y_{i}, z_{i}$ of the Solar System bodies in dimensionless coordinates, using the same procedure as for the analysis of the $b_{i}$ functions. Plots of the minimum value of $d_{\max }$ with respect to $N$, for fixed values of $T$, as well as tables with the values of the parameters for best analyses, can be found in ${ }^{6}$. 
Table 4. Values of the parameters for the best Fourier analyses of the $b_{i}$ functions for the Sun-(Earth+Moon) case. Note that, in this case, $J$-revolutions are sidereal years.

\begin{tabular}{cccccc}
\hline function & $T$ (days) & $T(J-\mathrm{rev})$ & $N$ & $p_{\max }$ & $d_{\max }$ \\
\hline$b_{1}$ & 142382.6 & 389.815 & 65536 & $4.95 \mathrm{E}-08$ & $4.40 \mathrm{E}-07$ \\
$b_{2}$ & 142382.6 & 389.815 & 65536 & $4.95 \mathrm{E}-08$ & $4.33 \mathrm{E}-07$ \\
$b_{3}$ & 112529.5 & 308.083 & 131072 & $2.28 \mathrm{E}-09$ & $2.68 \mathrm{E}-08$ \\
$b_{4}$ & 34698.8 & 94.998 & 4096 & $8.34 \mathrm{E}-06$ & $6.74 \mathrm{E}-05$ \\
$b_{5}$ & 34698.8 & 94.998 & 4096 & $1.75 \mathrm{E}-05$ & $1.26 \mathrm{E}-04$ \\
$b_{6}$ & 88935.7 & 243.488 & 262144 & $1.76 \mathrm{E}-08$ & $5.71 \mathrm{E}-07$ \\
$b_{7}$ & 34698.8 & 94.998 & 4096 & $1.36 \mathrm{E}-05$ & $9.17 \mathrm{E}-05$ \\
$b_{8}$ & 288422.1 & 789.642 & 524288 & $9.65 \mathrm{E}-08$ & $1.67 \mathrm{E}-06$ \\
$b_{9}$ & 88935.7 & 243.488 & 131072 & $9.71 \mathrm{E}-09$ & $3.19 \mathrm{E}-07$ \\
$b_{10}$ & 34698.8 & 94.998 & 4096 & $1.36 \mathrm{E}-05$ & $9.17 \mathrm{E}-05$ \\
$b_{11}$ & 70288.7 & 192.436 & 524288 & $2.35 \mathrm{E}-08$ & $2.38 \mathrm{E}-06$ \\
$b_{12}$ & 34698.8 & 94.998 & 4096 & $3.92 \mathrm{E}-06$ & $4.06 \mathrm{E}-05$ \\
$b_{13}$ & 34698.8 & 94.998 & 4096 & $1.34 \mathrm{E}-05$ & $9.47 \mathrm{E}-05$ \\
\hline
\end{tabular}

\section{Generation of simplified Solar System models}

In this section we will generate several simplified Solar System models using the Fourier approximations computed according to the previous section. The models obtained will be compared with other ones through the computation of residual accelerations.

\subsection{Adjustment using linear combinations of basic frequencies}

In order to give a more physical meaning to the results obtained from the Fourier analysis, we will write the computed frequencies as linear combinations, with integer coefficients, of basic ones. These basic frequencies can be identified as "natural" frequencies of the planetary and lunar theories. The introduction in the Fourier expansions of the basic frequencies will be the key point for the construction of models of motion with increasing dynamical complexity.

In principle, the basic frequencies will be extracted from the list of frequencies computed in the Fourier analysis and using the procedure explained below. Nevertheless, in some cases it can be convenient to introduce a fixed set of basic frequencies obtained by other means, for instance from an analytical lunar theory, and then write all the computed frequencies as 
linear combinations of the ones in this fixed set. Both approaches will be considered in what follows.

In order to better describe the procedure, we need two definitions. Assume that $\omega_{1}, \ldots, \omega_{n}$ is a set of basic frequencies and that a frequency $f$ can be written as $f=k_{1} \omega_{1}+\ldots+k_{n} \omega_{n}$ with $k_{1}, \ldots, k_{n}$ integer numbers, then we say that $f$ is a linear combination of $\omega_{1}, \ldots, \omega_{n}$ of order $k=\left|k_{1}\right|+\cdots+\left|k_{n}\right|$. We say that $f$ is a linear combination of $\omega_{1}, \ldots, \omega_{n}$ of order $k$ within tolerance $\epsilon>0$ if, for some $k_{1}, \ldots, k_{n}$ such that $k=\left|k_{1}\right|+\cdots+\left|k_{n}\right|$, we have

$$
\left|f-\left(k_{1} \omega_{1}+k_{2} \omega_{2}+\ldots+k_{n} \omega_{n}\right)\right|<\epsilon \text {. }
$$

A simple approach for the determination of basic frequencies is:

(1) Choose a maximum order of the linear combinations to be found.

(2) Choose a tolerance for the adjustment of frequencies as linear combination of basic ones.

(3) For each frequency, try out all the linear combinations of the current set of basic frequencies up the chosen maximum order.

(4) If any of the linear combinations fulfills the tolerance requirements, add the current frequency to the set of basic ones.

This procedure may add extra basic frequencies (and thus end up with a rationally dependent set) in some cases, for instance, if the current frequency is an integer divisor of one of the basic frequencies. To avoid this, instead of trying to adjust the current frequency as linear combination of the basic ones, we will try to adjust zero as linear combination of the current frequency and the basic ones. If we succeed to do this and the current frequency gets a coefficient different from \pm 1 , it may be necessary to divide some basic frequencies by this coefficient.

\subsection{Simplified models for the Sun-(Earth+Moon) system}

For the models to be developed in this section, and leaving aside the two primaries — Sun and Earth-Moon barycenter-, we will not consider any perturbing body in $\mathcal{S}^{*}$ As it will be shown, this provides rather accurate models and, at the same time, avoids the introduction of additional basic 
frequencies. In this way, in the equations of motion (5), we will only use the Fourier expansions of $b_{1}, \ldots, b_{13}$, and its general expression for the equations of motion will be

$$
\left\{\begin{array}{l}
\ddot{x}=b_{1}^{(i)}+b_{4}^{(i)} \dot{x}+b_{5}^{(i)} \dot{y}+b_{7}^{(i)} x+b_{8}^{(i)} y+b_{9}^{(i)} z+b_{13}^{(i)} \frac{\partial \Omega^{(i)}}{\partial x} \\
\ddot{y}=b_{2}^{(i)}-b_{5}^{(i)} \dot{x}+b_{4}^{(i)} \dot{y}+b_{6}^{(i)} \dot{z}-b_{8}^{(i)} x+b_{10}^{(i)} y+b_{11}^{(i)} z+b_{13}^{(i)} \frac{\partial \Omega^{(i)}}{\partial y} \\
\ddot{z}=b_{3}^{(i)}-b_{6}^{(i)} \dot{y}+b_{4}^{(i)} \dot{z}+b_{9}^{(i)} x-b_{11}^{(i)} y+b_{12}^{(i)} z+b_{13}^{(i)} \frac{\partial \Omega^{(i)}}{\partial z}
\end{array}\right.
$$

being

$$
\Omega^{(i)}=\frac{1-\mu_{E, M}}{\sqrt{\left(x-\mu_{E, M}\right)^{2}+y^{2}+z^{2}}}+\frac{\mu_{E, M}}{\sqrt{\left(x-\mu_{E, M}+1\right)^{2}+y^{2}+z^{2}}}
$$

The super-index $(i)$ that we have used for the $b_{j}^{(i)}, j=1, \ldots, 13$, and $\Omega^{(i)}$ functions will be used as a label for the different intermediate models, according to the number of basic frequencies retained in the Fourier expansions.

The numerical data obtained (see ${ }^{11}$ or contact the authors) suggests to take into consideration only the $b_{4}, b_{5}, b_{7}, b_{10}, b_{12}$ and $b_{13}$ functions. In addition to this simplification, we will not consider any Solar System body in Eq. (6), since, just using the $b_{i}$, we are already taking the Sun into account. Applying the procedure of Sect. 4.1 to the $b_{13}$ function, with tolerance $10^{-5}$ and maximum order 20 , we get the following four basic frequencies: $\nu_{1}=0.999992616, \nu_{2}=0.6255242728, \nu_{3}=0.9147445983$, $\nu_{4}=1.8313395538$. These four frequencies allow to adjust the frequencies of the Fourier analysis of the $b_{4}, b_{5}, b_{7}, b_{10}$ and $b_{12}$ functions. The results are given in Table 4.2.

According to this, for $i=1, \ldots, 4$ we define the model $\mathrm{SSSM}_{i}$ as the one given by Eq. (11), taking as $b_{j}^{(i)}$ the Fourier expansion of $b_{j}$ computed in Sect. 3.1, but keeping only the independent term and the frequencies that can be written as a linear combination of $\nu_{1}, \ldots, \nu_{i}$.

Once the different models have been produced, it is desirable to see if they are close or not the the "real" one, that is: the full equations of motion (Eqs. (5) and (6)) in which the time periodic functions, $b_{i}$ and $x_{i}, y_{i}, z_{i}$, are computed using the JPL ephemeris files. For these purposes we first select 
Table 5. Fourier analysis results of the dominant $b_{i}$ functions in the Earth-Sun system. The frequencies have been adjusted as linear combinations, $\sum k_{i} \nu_{i}$, of the four basic frequencies. The order of the linear combination, $k$, and the corresponding error are also displayed.

\begin{tabular}{|c|c|c|c|c|c|c|c|c|}
\hline Func & Frequency & Amplitude & Error & $k_{1}$ & $k_{2}$ & $k_{3}$ & $k_{4}$ & $\mathrm{k}$ \\
\hline \multirow[t]{7}{*}{$b_{4}$} & 0.00000000000 & $1.30000 \mathrm{E}-09$ & $0.00000 \mathrm{E}+00$ & 0 & 0 & 0 & 0 & 0 \\
\hline & 0.99999261980 & $3.33720 \mathrm{E}-02$ & $3.38800 \mathrm{E}-09$ & 1 & 0 & 0 & 0 & 1 \\
\hline & 1.99998564390 & $8.35280 \mathrm{E}-04$ & $4.11070 \mathrm{E}-07$ & 2 & 0 & 0 & 0 & 2 \\
\hline & 1.25103997640 & $3.93800 \mathrm{E}-05$ & $-8.56920 \mathrm{E}-06$ & 0 & 2 & 0 & 0 & 2 \\
\hline & 1.83134352170 & $3.40050 \mathrm{E}-05$ & $3.96790 \mathrm{E}-06$ & 0 & 0 & 0 & 1 & 1 \\
\hline & 0.91473091670 & $2.84920 \mathrm{E}-05$ & $-1.36820 \mathrm{E}-05$ & 0 & 0 & 1 & 0 & 1 \\
\hline & 2.99997409570 & $1.97160 \mathrm{E}-05$ & $-3.75350 \mathrm{E}-06$ & 3 & 0 & 0 & 0 & 3 \\
\hline \multirow[t]{7}{*}{$b_{5}$} & 0.00000000000 & $2.00000 \mathrm{E}+00$ & $0.00000 \mathrm{E}+00$ & 0 & 0 & 0 & 0 & 0 \\
\hline & 0.99999261700 & $6.67490 \mathrm{E}-02$ & $5.51530 \mathrm{E}-10$ & 1 & 0 & 0 & 0 & 1 \\
\hline & 1.99998563790 & $1.39230 \mathrm{E}-03$ & $4.05090 \mathrm{E}-07$ & 2 & 0 & 0 & 0 & 2 \\
\hline & 1.25103998380 & $6.69550 \mathrm{E}-05$ & $-8.56180 \mathrm{E}-06$ & 0 & 2 & 0 & 0 & 2 \\
\hline & 0.91475203530 & $6.12480 \mathrm{E}-05$ & $7.43700 \mathrm{E}-06$ & 0 & 0 & 1 & 0 & 1 \\
\hline & 1.83134663800 & $4.85690 \mathrm{E}-05$ & $7.08420 \mathrm{E}-06$ & 0 & 0 & 0 & 1 & 1 \\
\hline & 2.99997541480 & $3.01690 \mathrm{E}-05$ & $-2.43440 \mathrm{E}-06$ & 3 & 0 & 0 & 0 & 3 \\
\hline \multirow[t]{7}{*}{$b_{7}$} & 0.00000000000 & $1.00042 \mathrm{E}+00$ & $0.00000 \mathrm{E}+00$ & 0 & 0 & 0 & 0 & 0 \\
\hline & 0.99999261500 & $5.00800 \mathrm{E}-02$ & $-1.41660 \mathrm{E}-09$ & 1 & 0 & 0 & 0 & 1 \\
\hline & 1.99998562010 & $1.25350 \mathrm{E}-03$ & $3.87270 \mathrm{E}-07$ & 2 & 0 & 0 & 0 & 2 \\
\hline & 0.91475953220 & $4.82370 \mathrm{E}-05$ & $1.49340 \mathrm{E}-05$ & 0 & 0 & 1 & 0 & 1 \\
\hline & 1.25103999430 & $4.22440 \mathrm{E}-05$ & $-8.55130 \mathrm{E}-06$ & 0 & 2 & 0 & 0 & 2 \\
\hline & 2.99998010500 & $3.08040 \mathrm{E}-05$ & $2.25580 \mathrm{E}-06$ & 3 & 0 & 0 & 0 & 3 \\
\hline & 0.62552269280 & $2.71900 \mathrm{E}-05$ & $-1.58000 \mathrm{E}-06$ & 0 & 1 & 0 & 0 & 1 \\
\hline \multirow[t]{7}{*}{$b_{10}$} & 0.00000000000 & $1.00042 \mathrm{E}+00$ & $0.00000 \mathrm{E}+00$ & 0 & 0 & 0 & 0 & 0 \\
\hline & 0.99999261500 & $5.00800 \mathrm{E}-02$ & $-1.41650 \mathrm{E}-09$ & 1 & 0 & 0 & 0 & 1 \\
\hline & 1.99998562010 & $1.25350 \mathrm{E}-03$ & $3.87270 \mathrm{E}-07$ & 2 & 0 & 0 & 0 & 2 \\
\hline & 0.91475953220 & $4.82370 \mathrm{E}-05$ & $1.49340 \mathrm{E}-05$ & 0 & 0 & 1 & 0 & 1 \\
\hline & 1.25103999430 & $4.22440 \mathrm{E}-05$ & $-8.55130 \mathrm{E}-06$ & 0 & 2 & 0 & 0 & 2 \\
\hline & 2.99998010500 & $3.08040 \mathrm{E}-05$ & $2.25580 \mathrm{E}-06$ & 3 & 0 & 0 & 0 & 3 \\
\hline & 0.62552269280 & $2.71900 \mathrm{E}-05$ & $-1.58000 \mathrm{E}-06$ & 0 & 1 & 0 & 0 & 1 \\
\hline \multirow[t]{7}{*}{$b_{12}$} & 0.00000000000 & $-1.39300 \mathrm{E}-04$ & $0.00000 \mathrm{E}+00$ & 0 & 0 & 0 & 0 & 0 \\
\hline & 0.99999262330 & $1.66930 \mathrm{E}-02$ & $6.87550 \mathrm{E}-09$ & 1 & 0 & 0 & 0 & 1 \\
\hline & 1.99998564990 & $6.96230 \mathrm{E}-04$ & $4.17110 \mathrm{E}-07$ & 2 & 0 & 0 & 0 & 2 \\
\hline & 1.83134558880 & $3.11050 \mathrm{E}-05$ & $6.03500 \mathrm{E}-06$ & 0 & 0 & 0 & 1 & 1 \\
\hline & 1.25103987210 & $2.46550 \mathrm{E}-05$ & $-8.67350 \mathrm{E}-06$ & 0 & 2 & 0 & 0 & 2 \\
\hline & 2.99997235010 & $2.26070 \mathrm{E}-05$ & $-5.49910 \mathrm{E}-06$ & 3 & 0 & 0 & 0 & 3 \\
\hline & 0.91470513360 & $1.30450 \mathrm{E}-05$ & $-3.94650 \mathrm{E}-05$ & 0 & 0 & 1 & 0 & 1 \\
\hline \multirow[t]{7}{*}{$b_{13}$} & 0.00000000000 & $1.00042 \mathrm{E}+00$ & $0.00000 \mathrm{E}+00$ & 0 & 0 & 0 & 0 & 0 \\
\hline & 0.99999261640 & $5.00800 \mathrm{E}-02$ & $5.35290 \mathrm{E}-12$ & 1 & 0 & 0 & 0 & 1 \\
\hline & 1.99998562580 & $1.25340 \mathrm{E}-03$ & $3.93030 \mathrm{E}-07$ & 2 & 0 & 0 & 0 & 2 \\
\hline & 1.25104010020 & $4.71180 \mathrm{E}-05$ & $-8.44540 \mathrm{E}-06$ & 0 & 2 & 0 & 0 & 2 \\
\hline & 0.91474459830 & $4.67440 \mathrm{E}-05$ & $-4.82540 \mathrm{E}-11$ & 0 & 0 & 1 & 0 & 1 \\
\hline & 2.99997729050 & $3.07760 \mathrm{E}-05$ & $-5.58700 \mathrm{E}-07$ & 3 & 0 & 0 & 0 & 3 \\
\hline & 1.83133955380 & $2.81230 \mathrm{E}-05$ & $-9.85990 \mathrm{E}-12$ & 0 & 0 & 0 & 1 & 1 \\
\hline
\end{tabular}


a set of trajectories,

$$
\begin{array}{cccc}
\gamma_{z}: & \mathbb{R} & \longrightarrow & \mathbb{R}^{6} \\
& t & \longrightarrow & (\mathbf{r}(t), \dot{\mathbf{r}}(t)),
\end{array}
$$

along which the position, $\mathbf{r}(t)$, and velocity, $\dot{\mathbf{r}}(t)$, are known. We have done two kinds of selections. In the first one we have chosen for $\gamma_{z}$ a family of periodic halo orbits with different $z$-amplitudes; these orbits are true solutions of the RTBP ( see $^{1}$ for their computation) and cover a large set of solutions with very different sizes. Then, given two models to be compared, with differential equations $\ddot{\mathbf{r}}=f(\mathbf{r}, \dot{\mathbf{r}}, t)$ and $\ddot{\mathbf{r}}=g(\mathbf{r}, \dot{\mathbf{r}}, t)$, respectively, and given a trajectory, $\gamma_{z}$, which does not need to be a true solution of any of the two models, we compute the "mean relative residual acceleration over $\gamma^{\prime \prime}$ as

$$
\frac{1}{L} \int_{0}^{T} \frac{\left\|f\left(\gamma_{z}(s), t\right)-g\left(\gamma_{z}(s), t\right)\right\|}{\left\|g\left(\gamma_{z}(s), t\right)\right\|}\|\dot{\mathbf{r}}(s)\| d s,
$$

where $t$ is a fixed epoch (in dimensionless units) and $L$ is the length of the trajectory $\gamma_{z}$ (in configuration space).

For the second test the computations are similar except that we have taken instead of $\gamma_{z}(t)$ a set of points uniformly distributed around a large neighborhood of the equilibrium points. We have also required to their energy (Jacobi constant) to be in a certain interval around the value associated to the equilibrium points. The results obtained are analogous to the ones obtained for the halo orbits, and will not be given here.

In Table 4.2, we compare the models RTBP, $\mathrm{SSSM}_{1}$ and $\mathrm{SSSM}_{4}$ with the real Solar System using the residual acceleration test introduced above. We note that the $\mathrm{SSSM}_{4}$ model gives worse results than $\mathrm{SSSM}_{1}$. This is not a contradiction. Looking closer to Table 4.2 we can see that the maximum amplitude of the frequencies of $b_{4}, b_{5}, b_{7}, b_{10}$ and $b_{12}$ that are not multiple of $\nu_{1}$ is $6.695 \mathrm{E}-05$. Because of that, adding frequencies does not improve significantly the approximation of the $b_{i}$ functions, and in this way the structure of Eqs. (5) takes over the fact that the $b_{i}$ terms of $\mathrm{SSSM}_{4}$ are closer to the ones of the real Solar System than the corresponding terms of $\operatorname{SSSM}_{1}$.

Therefore, for the Sun-(Earth+Moon) case, we will give $\mathrm{SSSM}_{1}$ as simplified Solar System model. Note that this is a model with very few frequencies (just one) that significantly improves the RTBP. 
Table 6. Mean relative residual accelerations between several models and the real Solar System over selected halo orbits of the RTBP around $L_{2}$ in the Sun-(Earth+Moon) case.

\begin{tabular}{cccc}
\hline$z$-a. & RTBP & SSSM $_{1}$ & SSSM $_{4}$ \\
\hline 0.020000 & $3.446497 \mathrm{E}-02$ & $9.901526 \mathrm{E}-05$ & $8.905454 \mathrm{E}-04$ \\
0.024838 & $3.411184 \mathrm{E}-02$ & $9.779360 \mathrm{E}-05$ & $8.768670 \mathrm{E}-04$ \\
0.030846 & $3.366579 \mathrm{E}-02$ & $9.616913 \mathrm{E}-05$ & $8.589500 \mathrm{E}-04$ \\
0.038308 & $3.313580 \mathrm{E}-02$ & $9.416327 \mathrm{E}-05$ & $8.364166 \mathrm{E}-04$ \\
0.047575 & $3.254789 \mathrm{E}-02$ & $9.175134 \mathrm{E}-05$ & $8.092527 \mathrm{E}-04$ \\
0.059084 & $3.194355 \mathrm{E}-02$ & $8.895610 \mathrm{E}-05$ & $7.776813 \mathrm{E}-04$ \\
0.073376 & $3.137381 \mathrm{E}-02$ & $8.582841 \mathrm{E}-05$ & $7.420444 \mathrm{E}-04$ \\
0.091126 & $3.089082 \mathrm{E}-02$ & $8.236183 \mathrm{E}-05$ & $7.026421 \mathrm{E}-04$ \\
0.113169 & $3.053770 \mathrm{E}-02$ & $7.859979 \mathrm{E}-05$ & $6.597243 \mathrm{E}-04$ \\
0.140545 & $3.033772 \mathrm{E}-02$ & $7.450252 \mathrm{E}-05$ & $6.135638 \mathrm{E}-04$ \\
0.174543 & $3.028516 \mathrm{E}-02$ & $7.020714 \mathrm{E}-05$ & $5.643885 \mathrm{E}-04$ \\
0.216766 & $3.034115 \mathrm{E}-02$ & $6.579492 \mathrm{E}-05$ & $5.127031 \mathrm{E}-04$ \\
0.300000 & $3.047577 \mathrm{E}-02$ & $5.898080 \mathrm{E}-05$ & $4.323859 \mathrm{E}-04$ \\
\hline
\end{tabular}

\subsection{Simplified models for the Sun-(Earth+Moon) system}

For the Earth-Moon models to be developed in this section, and leaving aside the two primaries - Earth and Moon - the Sun will be the only perturbing body in $\mathcal{S}^{*}$. As it will be shown, this provides rather accurate models and, at the same time, avoids the introduction of additional basic frequencies.

In a rather accurate theory for the lunar motion, as the simplified Brown theory given in ${ }^{12}$, the fundamental parameters can be expressed in terms of five basic frequencies. In terms of cycles per lunar revolution, their numerical values are

- The mean longitude of the Moon, which is set equal to $\omega_{1}=1.0$.

- The mean elongation of the Moon from the Sun, $\omega_{2}=$ 0.925195997455093. This is the frequency of the time-dependent part of the Bicircular Problem (BCP) and the Quasi-Bicircular Problem (QBCP) mentioned in the Introduction.

- The mean longitude of the lunar perigee, $\omega_{3}=8.45477852931292 \cdot 10^{-3}$.

- The longitude of the mean ascending node of the lunar orbit on the ecliptic, $\omega_{4}=4.01883841204748 \cdot 10^{-3}$.

- The Sun's mean longitude of perigee, $\omega_{5}=3.57408131981537 \cdot 10^{-6}$. 
The value of $\omega_{5}$ is close to the lower frequencies computed in our Fourier expansions and, at the same time, is close to the precision we can expect in the determination of frequencies with the data used ${ }^{9}$. By these reasons and in order to have also a set of basic frequencies with astronomical meaning, we have adopted for the Earth-Moon models these frequencies as the basic set, instead of the ones provided by the procedure of Sect. 4.1.

This basic set of frequencies needs to be modified in order to give a sequence of models that successively improve the RTBP. The Fourier analysis results ${ }^{6}$ suggest to consider a new frequency basis $\nu_{1}, \ldots, \nu_{5}$, defined as:

- $\nu_{1}=\omega_{2}$, which is the main frequency of $b_{1}, b_{2}, x_{S}$ and $y_{S}$, so it can be considered the "main planar frequency", which is coherent with the fact that that it is also the frequency of the BCP and QBCP models $\left(\right.$ see $\left.^{13,1}\right)$.

- $\nu_{2}=\omega_{1}-\omega_{3}$, which allows to complete a first approximation of the largest functions among the $b_{i}$ and $x_{S}, y_{S}, z_{S}$.

- $\nu_{3}=\omega_{1}-\omega_{2}+\omega_{4}$, which is the main frequency of $b_{3}$.

- $\nu_{4}=\omega_{1}-\omega_{5}$, which is the first frequency of $x_{S}$ which cannot be expressed in terms of $\nu_{1}, \nu_{2}$, and

- $\nu_{5}=\omega_{5}-\omega_{2}$, which is the first frequency of $b_{3}$ that cannot be expressed in terms of $\nu_{1}, \nu_{2}, \nu_{3}, \nu_{4}$.

In order to check all the above statements, the full Fourier expansions can be found in ${ }^{11}$ or provided by the authors. The new basis verifies

$$
\left(\begin{array}{l}
\nu_{1} \\
\nu_{2} \\
\nu_{3} \\
\nu_{4} \\
\nu_{5}
\end{array}\right)=\left(\begin{array}{rrrrr}
0 & 1 & 0 & 0 & 0 \\
1 & 0 & -1 & 0 & 0 \\
1 & -1 & 0 & 1 & 0 \\
1 & 0 & 0 & 0 & -1 \\
0 & -1 & 0 & 0 & 1
\end{array}\right)\left(\begin{array}{l}
\omega_{1} \\
\omega_{2} \\
\omega_{3} \\
\omega_{4} \\
\omega_{5}
\end{array}\right) .
$$

Since the matrix in the above transformation is unimodular, $\left\{\nu_{i}\right\}_{i=1, \ldots, 5}$ is a valid basic set of frequencies.

The results for the residual accelerations are given in Table 4.3, using as test paths several halo orbits around the collinear equilibrium point $L_{2}$. As it has already been mentioned, the results with other trajectories, or other equilibrium points, give the same qualitative information. From this table, it becomes clear that the best one-frequency models that we can be used, 
Table 7. Mean residual accelerations between several models and the real Solar System over selected halo orbits of the RTBP around $L_{2}$ in the Earth-Moon case. The first column displays the $z$-amplitude of the halo orbit used as test orbit. The remaining columns show the mean residual acceleration between the corresponding model and the real Solar System over the test orbit.

\begin{tabular}{ccccccccc}
\hline$z$-a. & RTBP & BCP & QBCP & SSSM $_{1}$ & SSSM $_{2}$ & SSSM $_{3}$ & SSSM $_{4}$ & SSSM $_{5}$ \\
\hline 0.020 & 0.140126 & 0.146459 & 0.138580 & 0.365299 & 0.095769 & 0.010674 & 0.001374 & 0.000727 \\
0.025 & 0.136603 & 0.142856 & 0.135174 & 0.353302 & 0.093293 & 0.010388 & 0.001346 & 0.000720 \\
0.031 & 0.132882 & 0.139025 & 0.131578 & 0.340305 & 0.090590 & 0.010076 & 0.001315 & 0.000711 \\
0.038 & 0.129087 & 0.135080 & 0.127914 & 0.326550 & 0.087699 & 0.009744 & 0.001282 & 0.000702 \\
0.048 & 0.125352 & 0.131141 & 0.124312 & 0.312235 & 0.084643 & 0.009393 & 0.001247 & 0.000691 \\
0.059 & 0.121813 & 0.127324 & 0.120905 & 0.297505 & 0.081429 & 0.009024 & 0.001210 & 0.000678 \\
0.073 & 0.118614 & 0.123757 & 0.117835 & 0.282462 & 0.078045 & 0.008637 & 0.001171 & 0.000664 \\
0.091 & 0.115905 & 0.120571 & 0.115249 & 0.267173 & 0.074461 & 0.008229 & 0.001128 & 0.000646 \\
0.113 & 0.113823 & 0.117895 & 0.113283 & 0.251690 & 0.070634 & 0.007796 & 0.001081 & 0.000625 \\
0.141 & 0.112471 & 0.115836 & 0.112037 & 0.236056 & 0.066510 & 0.007331 & 0.001030 & 0.000598 \\
0.175 & 0.111872 & 0.114443 & 0.111533 & 0.220325 & 0.062042 & 0.006831 & 0.000973 & 0.000566 \\
0.217 & 0.111928 & 0.113663 & 0.111672 & 0.204551 & 0.057196 & 0.006292 & 0.000910 & 0.000526 \\
0.269 & 0.112400 & 0.113311 & 0.112201 & 0.188782 & 0.051978 & 0.005716 & 0.000840 & 0.000481 \\
0.300 & 0.112678 & 0.113200 & 0.112492 & 0.180899 & 0.049240 & 0.005417 & 0.000802 & 0.000456 \\
\hline
\end{tabular}

according to the residual acceleration criteria, are the $\mathrm{BCP}$ and the $\mathrm{QBCP}$. But, when we allow two or more frequencies, the models we get fit the JPL one much better. As it has been said, only the Sun has been taken into account in all the intermediate models. By adding additional Solar System bodies, the residual accelerations are of the same order of magnitude than the ones obtained just using the Sun. It is also clear that, from this point of view, there is not a significant improvement between the RTBP and the non-autonomous Bicircular and Quasi Bicircular models.

\section{Dynamical substitutes of the collinear libration points}

As it is well known, the RTBP has five equilibrium points: three of them $\left(L_{1}, L_{2}, L_{3}\right)$ are collinear with the primaries and the other two $\left(L_{4}\right.$ and $\left.L_{5}\right)$ form an equilateral triangle with them. Although the intermediate models introduced in the preceding section are close to the RTBP, they are non autonomous, so they do not have any critical point. If we consider the $\mathrm{SSSM}_{1}$ model, since it depends on only one frequency, it can be seen as a periodic perturbation of the RTBP so, under very general non-resonance conditions between the natural modes around the equilibrium points and the perturbing frequency, the libration points can be continued to periodic 
orbits of the model. These periodic orbits, which have the same period as the perturbation, are the dynamical substitutes of the equilibrium points. In this section we will show these substitutes for the three collinear equilibrium points for $\mathrm{SSSM}_{1}$, in the Earth-Moon system. For the other models, $\mathrm{SSSM}_{2}, \ldots, \mathrm{SSSM}_{5}$, as the perturbation is quasi-periodic, the corresponding substitutes will be also quasi-periodic solutions. The methodology for their efficient computation, as well as the results obtained, will appear elsewhere. The dynamical substitutes of the triangular points in the Earth-Moon system, for models close to the ones of this paper, have been studied in ${ }^{14},{ }^{13}$ and ${ }^{15}$ and will not be considered here.
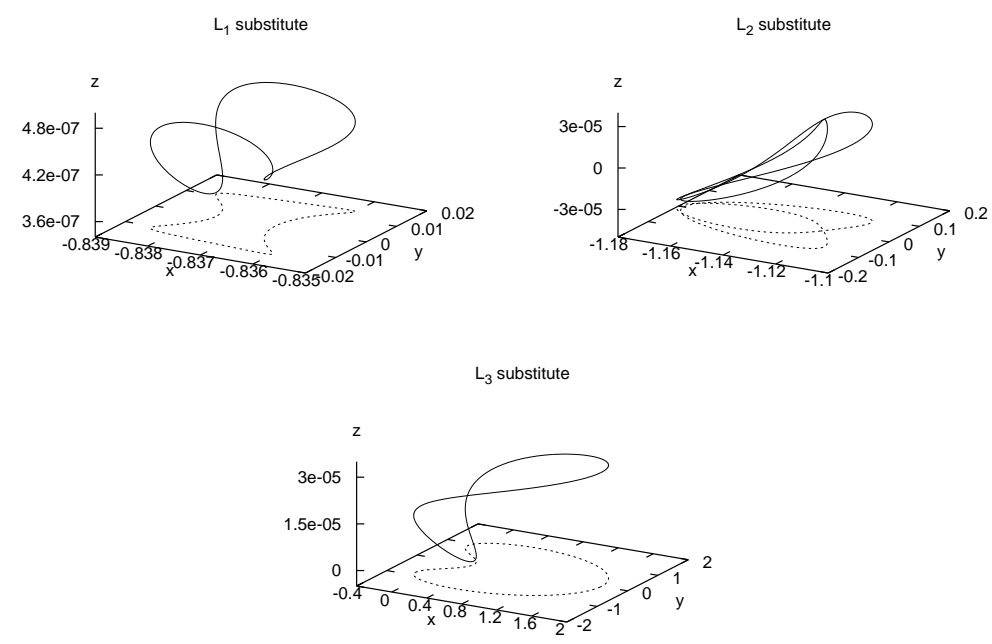

Fig. 3. Dynamical substitutes for the $\mathrm{SSSM}_{1}$ model of the three collinear equilibrium points.

The numerical computation of the periodic orbits of $\mathrm{SSSM}_{1}$ that substitute $L_{1}$ and $L_{3}$ has no problem and the results obtained are shown in Figure 3 . We can see that $L_{1}$ is replaced by a very small size periodic orbit and that the substitute of $L_{3}$ is also almost planar but rather large in the $(x, y)$-plane. The computation of the substitute of $L_{2}$, also displayed in Figure 3, requires more care. Mainly, we need to introduce a continuation parameter between the RTBP and $\mathrm{SSSM}_{1}$, so we consider the 1-parameter 
family of vector-fields which can be formally written as

$$
(1-\varepsilon) \mathrm{RTBP}+\varepsilon \mathrm{SSSM}_{1} .
$$

If $\varepsilon=0$ we get the RTBP and when $\varepsilon=1$ we get the desired final model $\mathrm{SSSM}_{1}$.

The dynamical substitutes of $L_{1,2,3}$ in $\mathrm{SSSM}_{2}$ are two-dimensional invariant tori. They can be computed as follows ${ }^{5,16}$ : the $\mathrm{SSSM}_{2}$ model can be written as

$$
\dot{x}=f(x, \nu t)
$$

where $\nu=\left(\nu_{1}, \nu_{2}\right)$ and $f$ is $2 \pi$-periodic in $\nu t$. We do not actually compute a 2D invariant torus but an invariant curve inside it. For that, we solve numerically for $\varphi$ the equation

$$
\varphi\left(\xi+\frac{2 \pi}{\nu_{1}}\right)=\phi_{2 \pi / \nu_{1}}^{(0, \xi)}(\varphi(\xi)), \quad \forall \xi \in[0,2 \pi)
$$

where $\phi_{t}^{\theta}(x)$ is the flow from time 0 to time $t$ of

$$
\dot{x}=f(x, \theta+\nu t)
$$

(which is not $\mathrm{SSSM}_{2}$ if $\theta \neq 0$ ). The geometrical torus is then

$$
\left\{\phi_{\theta_{1} / \nu_{1}}^{\left(0, \theta_{2}\right)}\left(\varphi\left(\theta_{2}\right)\right)\right\}_{\theta_{1}, \theta_{2} \in[0,2 \pi)}
$$

(see Fig. 4).

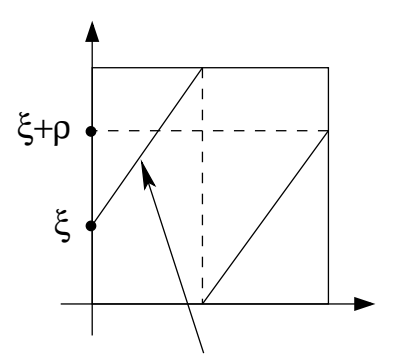

$(0, \xi)+v t$

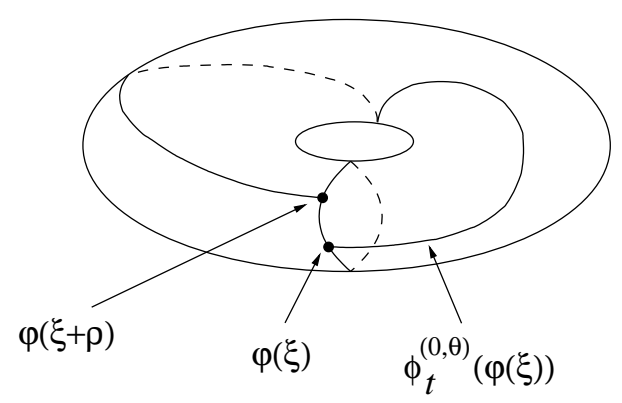

Fig. 4. Computation of two-dimensional invariant tori.

Using continuation techniques ${ }^{17}$, we can reach the substitutes of the collinear libration points in $\mathrm{SSSM}_{2}$ along the homothopy of models $(1-$ $\varepsilon) \operatorname{SSSM}_{1}+\varepsilon \mathrm{SSSM}_{2}$. As an example, we display in Figure 5 the results for the $L_{1}$ point of the Earth-Moon case. 

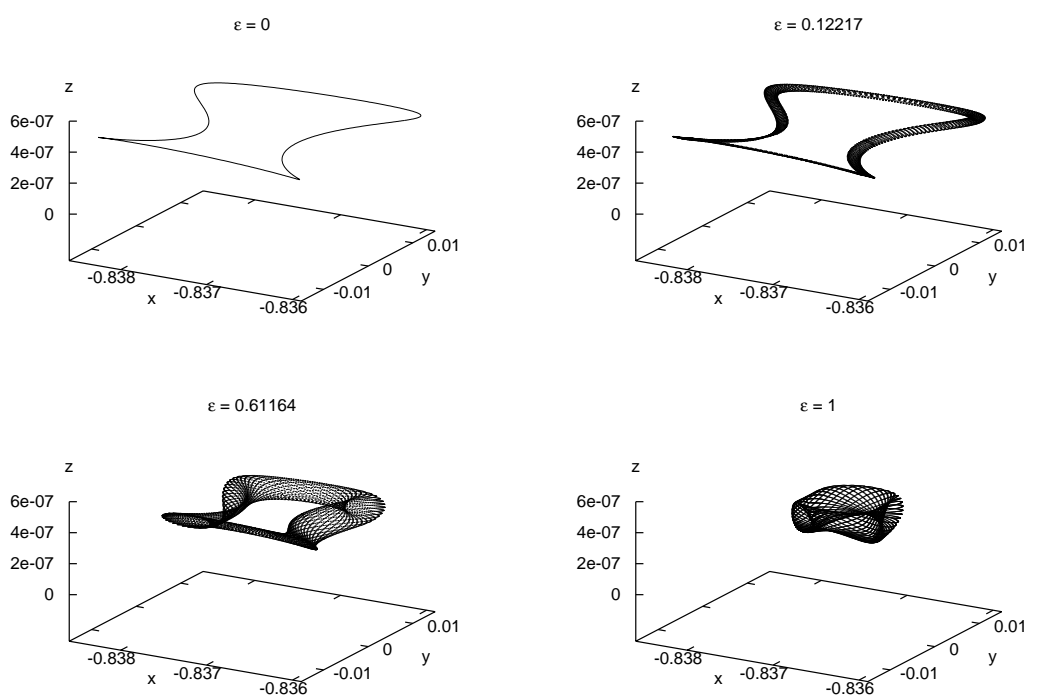

Fig. 5. Continuation from the dynamical substitute of $L_{1}$ in $\operatorname{SSSM}_{1}$ (a periodic orbit) to the dynamical substitute of $L_{1}$ in $\mathrm{SSSM}_{2}$ (a 2-dimensional torus), in the Earth-Moon case. The continuation is performed with respect to the parameter $\varepsilon$ along the family of models $(1-\varepsilon) \mathrm{SSSM}_{1}+\varepsilon \mathrm{SSSM}_{2}$.

\section{References}

1. G. Gómez, J. Llibre, R. Martínez, and C. Simó. Dynamics and Mission Design Near Libration Point Orbits - Volume 1: Fundamentals: The Case of Collinear Libration Points. World Scientific, 2001.

2. M.A. Andreu. The Quasibicircular Problem. PhD thesis, Dept. Matemàtica Aplicada i Anàlisi, Universitat de Barcelona, Barcelona, Spain, 1998. Available at http://www.maia.ub.es/dsg/.

3. K.C. Howell and J.J. Guzmán. Spacecraft trajectory design in the context of a coherent restricted four-body problem with application to the MAP mission. In 51st International Astronautical Congress, Rio de Janeiro, 2000.

4. F. Gabern and À. Jorba. A restricted four-body model for the dynamics near the Lagrangian points of the Sun-Jupiter system. Discrete Contin. Dyn. Syst. Ser. B, 1(2):143-182, 2001.

5. E. Castellà and À. Jorba. On the vertical families of two-dimensional tori near the triangular points of the bicircular problem. Celestial Mech. Dynam. Astronom., 76(1):35-54, 2000.

6. G. Gómez, J. J. Masdemont, and J. M. Mondelo. Solar system models with a 
selected set of frequencies. Astronomy \& Astrophysics, 390(2):733-749, 2002.

7. V. Szebehely. Theory of Orbits. Academic Press, 1967.

8. E.M. Standish. JPL Planetary and Lunar Ephemerides, de405/le405. Technical Report JPL IOM 314.10-127, NASA-Jet Propulsion Laboratory, 1998.

9. G. Gómez, J.M. Mondelo, and C. Simó. Refined Fourier Analysis Procedures. Preprint, 2001

10. E.O. Brigham. The Fast Fourier Transform. Prentice Hall, 1974.

11. J.M. Mondelo. Contribution to the Study of Fourier Methods for QuasiPeriodic Functions and the Vicinity of the Collinear Libration Points. PhD thesis, Dept. Matemàtica Aplicada i Anàlisi, Universitat de Barcelona, Barcelona, Spain, 2001. Available at http://www.maia.ub.es/dsg/.

12. P.R. Escobal. Methods of Astrodynamics. J. Wiley \& Sons, 1968.

13. C. Simó, G. Gómez, À. Jorba, and J. Masdemont. The Bicircular Model Near the Triangular Libration Points of the RTBP. In A. Roy and B. Steves, editors, From Newton to Chaos, pages 343-370. Plenum Press, 1995.

14. G. Gómez, À. Jorba, J. Masdemont, and C. Simó. Dynamics and Mission Design Near Libration Point Orbits - Volume 4: Advanced Methods for Triangular Points. World Scientific, 2001.

15. À. Jorba. A Numerical Study on the Existence of Stable Motions near the Triangular Points of the Real Earth-Moon System. Astron. Astrophys., 364(1):327-338, 2000.

16. À. Jorba. Private communication.

17. G. Gómez and J. M. Mondelo. The dynamics around the collinear equilibrium points of the RTBP. Phys. D, 157(4):283-321, 2001. 